\title{
Perfil de mulheres idosas segundo a ocorrência de quedas: estudo de demanda no Núcleo de Atenção ao Idoso da UnATI/UERJ
}

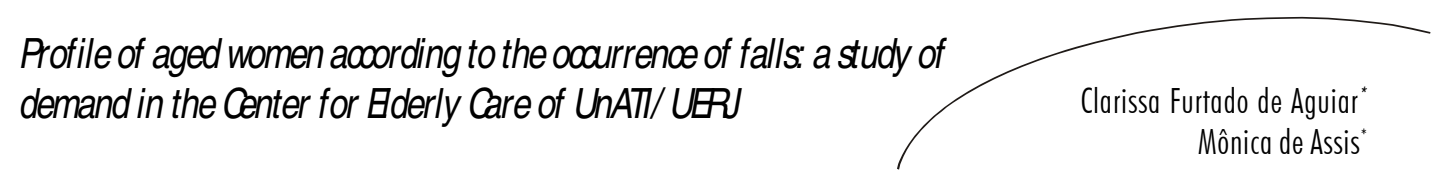

Resumo

A queda é um evento de elevada incidência após os 60 anos, sobretudo entre mulheres, e está associada ao aumento da morbi-mortalidade. O objetivo deste estudo foi caracterizar o perfil de mulheres idosas atendidas no Núcleo de Atenção ao Idoso da Universidade Aberta da Terceira Idade/UERJ no ano de 2007, em relação à ocorrência de quedas. Foram analisados 81 prontuários quanto a variáveis sociodemográficas, ocorrência e número de quedas no último ano, índice de massa corporal, número de doenças prévias relatadas, número de medicações em uso e testes de triagem positivos para déficit visual, alterações na mobilidade funcional, comprometimento da capacidade funcional, depressão e déficit cognitivo. Do total de idosas estudadas, $50(61,7 \%)$ relataram a ocorrência de uma ou mais quedas no último ano, com predominância na faixa etária de 70-79 anos. A maior parte do grupo que caiu apresentava disfunções nutricionais $(60,4 \%)$, quatro ou mais morbidades (76\%) e sintomas sugestivos de depressão (62\%). A média de quedas foi maior no grupo com capacidade funcional intermediária. Conclui-se que é importante incorporar a avaliação do risco de quedas por meio dos testes funcionais na rotina assistencial do idoso, para evidenciar e monitorar, de forma simples e com baixo custo, alterações que demandem intervenções interdisciplinares preventivas, assistenciais e de reabilitação, que contribuam para uma maior expectativa de vida saudável e recuperação ou manutenção da qualidade de vida de idosas que sofreram quedas.

Universidade do Estado do Rio de Janeiro. Curso de Especialização em Geriatria e Gerontologia, Universidade Aberta da Terceira Idade. Rio de Janeiro, RJ, Brasil

Correspondência / Correspondence

Clarissa Furtado de Aguiar

E-mail: clarissa.f.aguiar@gmail.com

\author{
Palavras-chave: \\ Idoso. Acidentes \\ por Quedas. \\ Assistência Integral \\ à Saúde. Prevenção \\ $\&$ Controle. \\ Medição de Risco. \\ Reabilitação. \\ Mulheres.
}




\section{Abstract}

The fall is an event of high incidence after 60 years, especially among women, and is associated with increased morbidity and mortality. This study aimed to characterize the profile of aged women treated at the outpatient unit of the Open University for Studies on the Elderly / UERJ in the year of 2007, in relation to the occurrence of falls. Records were collected on 81 anamneses of elderly women on socio-demographic variables, occurrence and number of falls in the last year, Body Mass Index, number of previous diseases reported, number of medications in use, and positive screening tests for visual acuity, changes in functional mobility, impairment of functional capacity, depression and cognitive impairment. From the total of elderly

Keywords: Aged. Accidental Falls. Comprehensive Health Care. Prevention \& Controls. Risk Assessment. Rehabilitation. Women. woman studied, $50(61.7 \%)$ reported the occurrence of one or more falls in the last year, predominantly in the age group of 70-79 years old. Most of the group which fell presented nutritional disorders (60.4\%), four or more morbidities (76\%) and symptoms of depression (62\%). The falls average was higher in the group with intermediate functional capacity. It follows that it is important to incorporate the assessment of the risk of falls by functional tests in the routine care of the elderly to highlight and monitor, by a simple form and with low cost, changes that demand interdisciplinary preventive interventions, caring and rehabilitation, contributing to a more healthy life expectancy and recovering or maintaining a higher quality of life to elderly people which suffered falls.

\section{INTRODUÇ̃̃O}

O envelhecimento populacional é hoje uma realidade mundial. ${ }^{1}$ No Brasil, os principais indicadores demográficos responsáveis por este processo são o declínio gradativo e contínuo das taxas de mortalidade infantil e de fecundidade, com consequente aumento da expectativa de vida. ${ }^{2}$

Segundo dados do IBGE, 2 em 2005 a esperança de vida ao nascer no Brasil era de 72 anos para ambos os sexos, com uma diferença de 7,5 anos a mais para as mulheres. A projeção para 2030 é de 78,3 anos, tendo ainda as mulheres uma expectativa de vida superior à dos homens, de 6,9 anos.
Esta diferença caracteriza a feminização da velhice no Brasil. Conforme indicadores demográficos no ano de 2005 , enquanto a proporção de idosos na população brasileira era de 9,2\%, e na capital do Rio de Janeiro, de $13,7 \%$, as mulheres idosas representavam respectivamente $10 \%$ e $15,8 \%$ da população. ${ }^{3}$

O envelhecimento tem sido associado a uma prevalência aumentada de doenças crônicas e incapacidade. ${ }^{4} \mathrm{~A}$ queda é um evento de elevada incidência na faixa etária acima dos 60 anos, sobretudo entre as mulheres, ${ }^{1}$ e está associada a significativas morbidade e mortalidade. ${ }^{4}$ De acordo com a Sociedade Brasileira de Geriatria e Gerontologia, ${ }^{5}$ a in- 
cidência de quedas chega a atingir $32 \%$ dos indivíduos de 64 a 74 anos; $35 \%$ na faixa de 75 a 84 anos e até $51 \%$ em idosos acima de 85 anos.

A queda pode ser definida como evento não intencional que tem como resultado a mudança de posição do indivíduo para um nível mais baixo, em relação a sua posição inicial. ${ }^{6}$

Diversos fatores de risco podem ser atribuídos ao risco elevado de quedas em indivíduos idosos. Os fatores intrínsecos são as alterações fisiológicas decorrentes do processo de envelhecimento no controle da postura e da marcha, que retardam respostas quanto à velocidade e à precisão, atrasando a seletividade dessas respostas e, consequentemente, inibindo as reações automáticas de equilíbrio. ${ }^{4}$ Entre estas mudanças, estão o declínio funcional neurológico e músculo-esquelético. Os déficits sensoriais, isto é, da visão, da propriocepção e do sistema vestibular, diminuem as pistas ambientais e predispõem às quedas. A presença de múltiplas comorbidades também eleva o risco de quedas. ${ }^{1}$ Idosos com déficit cognitivo, depressão e/ou ansiedade podem apresentar alteração do nível de atenção e/ ou desenvolver apraxia na marcha. ${ }^{1}$ Idosos com demência apresentaram um risco aproximadamente $80 \%$ maior de quedas seguidas de fraturas graves. ${ }^{8}$ A polifarmácia, caracterizada pelo uso de cinco ou mais drogas associadas, é outro fator de risco. ${ }^{5}$ Vários estudos indicam a associação entre o uso de medicações e quedas, em decorrência de inúmeros mecanismos de ação diretos e indiretos das drogas utilizadas pelos idosos. ${ }^{9}$
Os fatores extrínsecos estão relacionados aos riscos ambientais. ${ }^{4}$

Cerca de $30 \%$ dos idosos brasileiros relatam no mínimo uma queda ao ano, com maior frequência entre as mulheres. ${ }^{5}$ Aproximadamente metade sofre duas ou mais quedas. ${ }^{10}$ Em estudo conduzido em 1996 com idosos que frequentavam a Universidade Aberta da Terceira Idade da Universidade Estadual do Rio de Janeiro, Rozenfeld ${ }^{11}$ encontrou uma incidência de $38 \%$ de pelo menos uma queda no último ano.

Segundo Perracini $\&$ Ramos, ${ }^{10}$ as quedas têm como consequências possíveis as fraturas e/ou contusões com risco direto ou indireto de morte, o medo de cair novamente, a restrição de atividades, o declínio na saúde e o aumento do risco de institucionalização. ${ }^{5}$ Os idosos vítimas de quedas, além das complicações físicas e/ou psicológicas, ${ }^{4}$ apresentam-se de modo mais crítico inicialmente, necessitam mais frequentemente de internação hospitalar e representam grande proporção dos pacientes internados em unidades de tratamento intensivo, ${ }^{12} \mathrm{e}$ consomem mais recursos com cuidados de saúde do que pacientes de qualquer outra faixa etária. ${ }^{13,14}$

No Brasil, segundo dados do Sistema de Informação Hospitalar (SIH/SUS), em 2005 as quedas foram responsáveis por $54 \%$ das causas externas de internações hospitalares em indivíduos com 60 anos ou mais. ${ }^{3}$

Nos Estados Unidos, grande parte das quedas em idosos é associada com custos 
econômicos substanciais, para indivíduos, sociedade e o sistema de cuidados médicos de saúde. As despesas médicas foram 2-3 vezes maiores entre as mulheres idosas, quando comparado aos homens. ${ }^{14}$

O aumento acelerado da população idosa brasileira traz uma preocupação quanto à implementação de políticas públicas. ${ }^{15} \mathrm{~A}$ previsão é de aumento progressivo da demanda por serviços de saúde, paralelamente ao aumento relativo da população economicamente inativa, determinando aumento considerável dos gastos públicos. ${ }^{13}$ Essa mudança no perfil epidemiológico destaca a importância de novos modelos assistenciais capazes de melhorar a relação custo-benefício da prevenção, da detecção precoce e do tratamento das morbidades associadas à ocorrência de quedas. ${ }^{1}$

A Política Nacional de Saúde da Pessoa Idosa ${ }^{15}$ fundamenta a ação do setor saúde na atenção integral e tem por finalidade recuperar, manter e promover a autonomia e a independência do idoso. Aprovada em 2006, essa política prevê o acesso dos idosos aos serviços e às ações voltadas à promoção, proteção e recuperação da saúde.

É necessário conhecer o perfil da população idosa que cai, com o objetivo de instrumentalizar os profissionais e aprimorar as práticas assistenciais.

O objetivo deste estudo foi caracterizar o perfil de mulheres idosas de um ambulatório público especializado em relação à ocorrência de quedas. A identificação de idosas com um perfil de risco elevado de queda visa a possibilitar o planejamento de intervenções preventivas e/ou de reabilitação apropriadas. $O$ benefício às idosas se estende a suas famílias pela potencial redução de cuidados e custos no tratamento, como também à rede pública de saúde, pela consequente diminuição de gastos gerados pelas quedas.

\section{METODOLOGIA}

O presente trabalho é um estudo descritivo sobre o perfil de mulheres idosas, usuárias de um ambulatório especializado, segundo a ocorrência de quedas.

Os dados foram colhidos dos prontuários de mulheres com idade igual ou superior a 60 anos atendidas no Núcleo de Atenção ao Idoso (NAI), ambulatório vinculado à Universidade Aberta da Terceira Idade (UnATI), da Universidade do Estado do Rio de Janeiro (UERJ), no período de janeiro a dezembro de 2007. Esta unidade recebe idosos por demanda espontânea ou encaminhados por ambulatórios das especialidades médicas do Hospital Universitário Pedro Ernesto.

Foram analisados 81 prontuários quanto às seguintes variáveis: idade, escolaridade, ocorrência e número de quedas no último ano, peso e altura para o cálculo do estado nutricional, número de doenças prévias relatadas, número de medicações em uso, e testes de triagem positivos para déficit visual, alterações na mobilidade funcio- 
nal, comprometimento da capacidade funcional, depressão e déficit cognitivo.

A análise foi desagregada por faixas etárias, para observar possíveis diferenças de perfis entre os subgrupos.

Para a análise do estado nutricional das idosas, adotou-se o Índice de Massa Corporal (IMC), calculado pela fórmula peso (em $\mathrm{kg}$ ) dividido pelo quadrado da altura (em metros). ${ }^{16}$ Valores de IMC acima ou abaixo da faixa de normalidade estão associados a uma maior morbi-mortalidade. ${ }^{17}$ Adotou-se a classificação recomendada pela OMS,${ }^{18}$ seguindo o nível de corte específico para a população idosa, que estabelece como baixo peso o IMC $\leq 22 \mathrm{~kg} / \mathrm{m}^{2}$ (baixo peso), adequado quando $>22 \mathrm{e}<27 \mathrm{~kg} /$ $\mathrm{m}^{2}$ (eutrófico), e sobrepeso o IMC $\geq 27,0$ $\mathrm{kg} / \mathrm{m}^{2}{ }^{19}$ A diferença em relação aos pontos de corte adotados para adultos deve-se às alterações fisiológicas nos idosos, como o declínio da estatura, redução do peso relacionada à perda hídrica e de massa muscular e alterações ósseas, entre outras. ${ }^{20}$

A acuidade visual foi avaliada pelo cartão de Jaeger. ${ }^{17} \mathrm{O}$ cartão é colocado a cerca de 35 $\mathrm{cm}$ da idosa, mantida a correção visual durante o exame. A visão deve ser testada em cada olho em separado e depois em conjunto. A incapacidade de ler além de 20/40 no cartão de Jaeger indica disfunção visual. ${ }^{20}$

O equilíbrio foi verificado através do Timed Up \& Go (TUG), ${ }^{21}$ teste de equilíbrio frequentemente utilizado para examinar a mobilidade funcional em idosos. Este avalia a velocidade com que o indivíduo consegue realizar a tarefa de se levantar de uma cadeira, andar três metros, retornar e sentar. Adotou-se o ponto de corte proposto por Rockwood et al., ${ }^{22}$ no qual um tempo de execução do teste superior a 12 segundos está relacionado com um maior risco de quedas.

A capacidade funcional foi verificada com a aplicação da Escala de Atividades Básicas de Vida Diária (ABVDs) de Katz, ${ }^{23}$ amplamente incluída em avaliações geriátricas multidimensionais. Mede a habilidade da pessoa em desempenhar suas atividades básicas cotidianas, ${ }^{1}$ e classifica o sujeito como independente (A e B), com funcionalidade intermediária (C, D e E) ou dependente $\left(\mathrm{F}\right.$ e G)${ }^{24}$

A EDG visa a detectar estados sugestivos de depressão por meio do autorrelato de sintomas ligados à doença. Consiste em um questionário com 15 perguntas objetivas, de fácil entendimento e aplicação. $\mathrm{O}$ escore sugere depressão a partir de cinco pontos. ${ }^{25}$

O possível comprometimento cognitivo foi avaliado através do Mini-Exame do Estado Mental (MEEM), ${ }^{26}$ considerando-se os escores recomendados por Lourenço \& Veras, ${ }^{27}$ sendo $18 / 19$ o melhor ponto de corte para analfabetos e $24 / 25$, para idosos escolarizados. Os 11 itens examinam orientação temporal e espacial, memória de curto prazo (imediata ou atenção) e de evocação, cálculo, praxia e habilidades de linguagem e viso-espaciais. ${ }^{1}$ 
As informações foram digitadas em banco de dados e analisadas através de distribuições percentuais no Microsoft Excel.

\section{RESULTADOS}

No ano de 2007 foram inseridas 81 idosas no ambulatório do NAI. A média de idade foi 74,3 $( \pm 6,7)$ anos e a mais velha tinha 88 anos. Aproximadamente metade das idosas do estudo estava na faixa etária de 70 a 79 anos. A maioria possuía um ou mais anos de escolaridade, representando $78 \%$ do grupo que sofreu quedas, e $75,3 \%$ de todas as idosas.

A morbidade relatada com mais frequência foi hipertensão arterial sistêmica $(65,4 \%)$, seguida por tonteira $(54,3 \%)$, dor limitante $(48,1 \%)$, dislipidemia $(40,7 \%)$, incontinência urinária $(24,7 \%)$, cardiopatia $(23,5 \%)$, e diabetes $(22,2 \%)$. Do total de idosas estudadas, $50(61,7 \%)$ relataram a ocorrência de uma ou mais quedas no último ano (tabela 1).

Tabela 1 - Caracterização sociodemográfica, principais morbidades e ocorrência de quedas em mulheres idosas atendidas no ambulatório do NAI - UnATI/UERJ, $2007(\mathrm{n}=81)$. Rio de Janeiro, RJ, 2007.

\begin{tabular}{lcc}
\hline Variável & $\mathrm{n}(\%)$ \\
\hline Faixa etária & $60-69$ & $17(21)$ \\
& $70-79$ & $40(49,4)$ \\
& $80 \mathrm{ou}+$ & $24(29,6)$
\end{tabular}

Principais Morbidades

$\begin{array}{rr}\text { HAS } & 53(65,4) \\ \text { Tonteira } & 44(54,3) \\ \text { Dor limitante } & 39(48,1) \\ \text { Dislipidemia } & 33(40,7) \\ \text { Incontinência urinária } & 20(24,7) \\ \text { Cardiopatia } & 19(23,5) \\ \text { DM } & 18(22,2)\end{array}$

Ocorrência de quedas no ano anterior

Sim $\quad 50(61,7)$

Não $\quad 31(38,3)$ 
O grupo que sofreu quedas foi predominantemente o de mulheres na faixa etária de 70 a 79 anos (52\%), seguido das que tinham 80 anos ou mais ( $32 \%$ ) e das que tinham entre
60 e 69 anos (16\%). Como mostra a tabela 2, as idosas na faixa etária de 70 a 79 anos foram também as que apresentaram o maior número médio de quedas no último ano $(3,9)$.

Tabela 2 - Ocorrência e número médio de quedas no ano anterior por faixa etária, em mulheres idosas atendidas no ambulatório do NAI - UnATI/UERJ, 2007 ( $\mathrm{n}=50)$. Rio de Janeiro, RJ, 2007.

\begin{tabular}{ccc}
\hline Faixa etária & Quedas no último ano $\mathrm{n}(\%)$ & $\begin{array}{c}\text { Média de } \\
\text { quedas }( \pm \mathrm{dp})\end{array}$ \\
\hline $60-69$ & $8(16)$ & $3,1(2,9)$ \\
$70-79$ & $26(52)$ & $3,9(4,9)$ \\
$80 \mathrm{ou}+$ & $16(32)$ & $1,5(1,2)$ \\
Total & $50(100)$ & $3(3,9)$ \\
\hline
\end{tabular}

A tabela 3 apresenta a ocorrência de quedas no ano anterior, segundo o IMC e a faixa etária. A maior parte do grupo que caiu apresentava disfunções nutricionais
$(60,4 \%)$, isto é, baixo peso ou sobrepeso, segundo a classificação do IMC para idosos, sendo esta situação mais expressiva na faixa etária de 70 a 79 anos $(35,4 \%)$.

Tabela 3 - Ocorrência de quedas no ano anterior, segundo o Índice de Massa Corporal (IMC) e faixa etária, em mulheres idosas atendidas no ambulatório do NAI - UnATI/ UERJ, $2007(\mathrm{n}=$ 48)*. Rio de Janeiro, RJ, 2007.

\begin{tabular}{|c|c|c|c|c|c|}
\hline \multirow[t]{2}{*}{ Variável } & & \multicolumn{4}{|c|}{ Quedas no ano anterior $\mathrm{n}(\%)$} \\
\hline & & $60-69$ & $70-79$ & $80 \mathrm{ou}+$ & Total \\
\hline \multicolumn{6}{|l|}{ IMC } \\
\hline & Baixo peso & - & $4(8,3)$ & $2(4,2)$ & $6(12,5)$ \\
\hline & Eutrofia & $4(8,3)$ & $7(14,6)$ & $8(16,7)$ & $19(39,6)$ \\
\hline & Sobrepeso & $4(8,3)$ & $13(27,1)$ & $6(12,5)$ & $23(47,9)$ \\
\hline Total & & $8(16,7)$ & $24(50)$ & $16(33,3)$ & $48(100 \%)$ \\
\hline
\end{tabular}

* Dois prontuários não continham os dados antropométricos necessários para o cálculo do IMC. 
A tabela 4 mostra que $76 \%$ das idosas que sofreram quedas apresentavam quatro ou mais morbidades, e que $70 \%$ faziam uso de uma a quatro medicações. O número de morbidades variou entre uma e 11 e o de medicações entre nenhuma e 12.

Tabela 4 - Ocorrência de quedas no ano anterior, segundo o número de morbidades, medicações em uso e faixa etária, em mulheres idosas atendidas no ambulatório do NAI UnATI/UERJ, 2007 ( $\mathrm{n}=50)$. Rio de Janeiro, RJ, 2007.

\begin{tabular}{lrcccc}
\hline Variável & \multicolumn{4}{c}{ Quedas no ano anterior n(\%) } \\
\cline { 3 - 6 } & & $60-69$ & $70-79$ & $80 \mathrm{ou}+$ & Total \\
\hline Número de Morbidades & Até 1 & - & $2(4)$ & - & $2(4)$ \\
& $2-3$ & - & $6(12)$ & $4(8)$ & $10(20)$ \\
& $4 \mathrm{ou}+$ & $8(16)$ & $18(36)$ & $12(24)$ & $38(76)$ \\
Medicações em uso & & & & & \\
& Nenhuma & - & $2(4)$ & $1(2)$ & $3(6)$ \\
& $1-4$ & $5(10)$ & $18(36)$ & $12(24)$ & $35(70)$ \\
Total & 5 ou + & $3(6)$ & $6(12)$ & $3(6)$ & $12(24)$ \\
& & $8(16)$ & $26(52)$ & $16(32)$ & $50(100)$ \\
\hline
\end{tabular}

Na tabela 5, estão apresentadas as variáveis físico-funcionais das idosas que sofreram quedas. Observou-se a presença de déficit visual em 26 idosas (52\%) e de propensão a quedas em $54 \%$ do grupo, verificada pela realização do TUG. O grupo que caiu era predominantemente composto de mulheres idosas independentes, e o número médio de quedas foi similar entre as idosas dependentes $(2 \pm 1,4)$ e as independentes $(2,9 \pm 3,2)$, e ligeiramente maior entre as idosas com funcionalidade intermediária $(3,4 \pm 6,5)$. 
Tabela 5 - Ocorrência de quedas no ano anterior, segundo variáveis físico-funcionais e faixa etária, em mulheres idosas atendidas no ambulatório do NAI - UnATI/UERJ, 2007 $(\mathrm{n}=50)$. Rio de Janeiro, RJ, 2007.

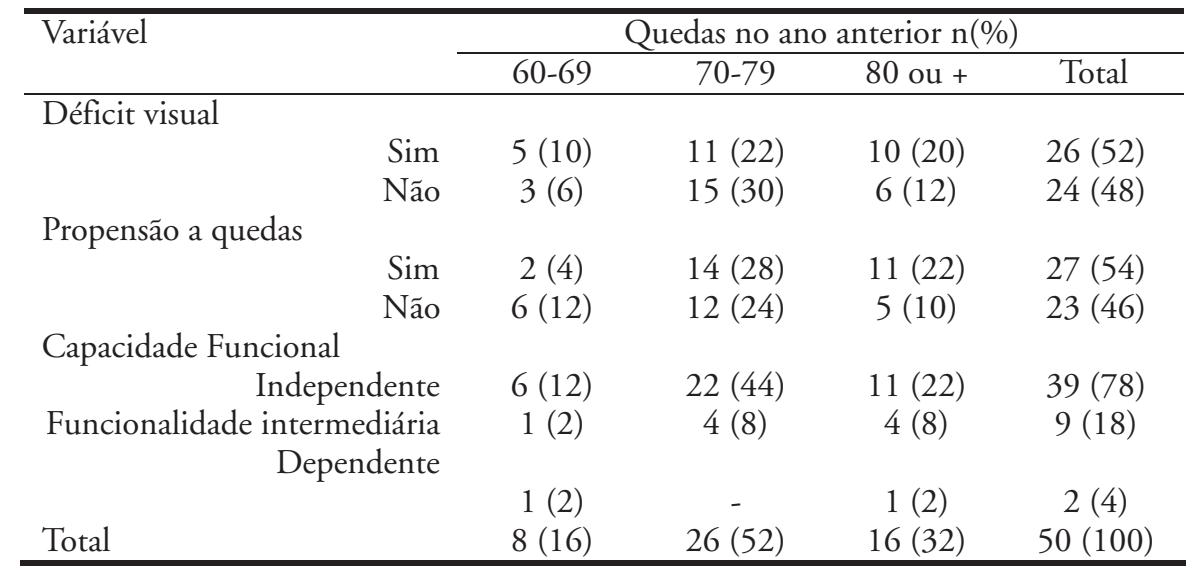

A distribuição das idosas que tiveram quedas segundo as variáveis psicocognitivas e faixa etária é apresentada na tabela 6. A EDG identificou 31 (62\%) idosas com sintomas sugestivos de depressão. Na faixa etária de 60 a 69 anos, sete entre oito idosas que caíram apresentaram sintomas depressivos. Já o déficit cognitivo estava presente em $52 \%$ delas, as quais apresentaram uma média de $3,2( \pm 4,6)$ quedas no ano anterior, contra 2,7 $( \pm 2,9)$ quedas no grupo sem indicações de distúrbio cognitivo. A ocorrência de comprometimento cognitivo foi semelhante em todas as faixas etárias.

\section{DISCUSSÃO}

Os resultados mostram maior susceptibilidade para quedas nas idosas com mais de 70 anos.

Entre as que sofreram quedas, predominaram idosas com disfunções nutricionais, caracterizadas por sobrepeso ou magreza. Alguns estudos têm demonstrado a associação de disfunções nutricionais em idosos com significativa morbidade e mortalidade ${ }^{19}$ e com maior propensão a quedas. ${ }^{28}$ A predominância de sobrepeso entre 
Tabela 6 - Ocorrência de quedas no ano anterior, segundo variáveis psicocognitivas e faixa etária, em mulheres idosas atendidas no ambulatório do NAI - UnATI/UERJ, 2007 ( $n=50)$. Rio de Janeiro, RJ, 2007.

\begin{tabular}{|c|c|c|c|c|}
\hline \multirow[t]{2}{*}{ Variável } & \multicolumn{4}{|c|}{ Quedas no ano anterior $\mathrm{n}(\%)$} \\
\hline & $60-69$ & $70-79$ & $80 \mathrm{ou}+$ & Total \\
\hline \multicolumn{5}{|c|}{ Sintomas depressivos } \\
\hline Sim & $7(14)$ & $17(34)$ & $7(14)$ & $31(62)$ \\
\hline Não & $1(2)$ & $9(18)$ & $9(18)$ & $19(38)$ \\
\hline \multicolumn{5}{|c|}{ Déficit cognitivo } \\
\hline Sim & $3(6)$ & $15(30)$ & $8(16)$ & $26(52)$ \\
\hline Não & $5(10)$ & $11(22)$ & $8(16)$ & $24(48)$ \\
\hline Total & $8(16)$ & $26(52)$ & $16(32)$ & $50(100)$ \\
\hline
\end{tabular}

as idosas deste estudo confirma a tendência verificada nas últimas décadas na população brasileira, ${ }^{29}$ sobretudo nas camadas socioeconômicas intermediárias e baixas, situação que sinaliza um problema de saúde pública e a necessidade de adoção de políticas preventivas.

A hipertensão foi a doença crônica mais frequentemente relatada, confirmando observações de outros estudos com população idosa. ${ }^{30}$ Com certa frequência, as morbidades de características crônicas, mais comuns entre os idosos, evoluem com algum grau de perda da capacidade funcional, que pode estar associado à dependência física e à imobilidade. Grande parte do grupo de idosas que sofreu uma ou mais quedas no último ano apresentava quatro ou mais morbidades. Tinetti ${ }^{31}$ relatou que as morbidades crônicas contribuem para a ocorrência de quedas em idosos, e que o risco de quedas aumenta linearmente com o número de alterações existentes.
A maioria das idosas que caíram tomava entre uma e quatro medicações, seguidas de cinco ou mais. Em decorrência do envelhecimento fisiológico, várias alterações interferem na farmacocinética e farmacodinâmica dos medicamentos. ${ }^{32}$ Segundo Tinetti, ${ }^{31}$ associa-se um maior número de prescrições medicamentosas com maior morbidade e maior média de quedas entre as idosas. Alguns autores afirmam que quanto maior o número de doenças identificadas, maior a lista de prescrições, e que o número de efeitos colaterais tende a aumentar com o número de medicações. ${ }^{32}$ Drogas como sedativos/ansiolíticos, antidepressivos e agentes cardiovasculares, podem predispor quedas em idosos em virtude dos efeitos colaterais como hipotensão postural, arritmias, sedação excessiva, alterações no equilíbrio, na marcha e no estado de alerta cognitivo. ${ }^{9}$

A ocorrência de quedas foi semelhante entre as idosas com e sem déficit visual. Em outros estudos, ${ }^{33,34}$ observou-se maior com- 
prometimento do equilíbrio em idosos com déficit visual, predispondo-os à maior ocorrência de quedas e suas consequências.

O tempo gasto para realização do TUG está diretamente associado ao nível de mobilidade funciona ${ }^{21} \mathrm{e}$ relacionado à velocidade da marcha e ao equilíbrio. De acordo com Vellas et al., ${ }^{35}$ o risco de quedas em mulheres idosas é mais elevado quando associado ao déficit de mobilidade funcional. Neste trabalho, a avaliação através do TUG mostrou que muitas idosas que caíram no último ano têm propensão a novas quedas, pois apresentam baixa velocidade de marcha e instabilidade postural.

A maioria dos idosos do estudo apresentou-se independente na realização das suas atividades diárias. Observou-se, no entanto, que a média de quedas foi discretamente maior no grupo de idosas com capacidade funcional intermediária. Idosos com comprometimento das habilidades para desempenhar $\mathrm{ABVD}$ s são mais vulneráveis às quedas, ${ }^{6}$ bem como ao risco aumentado de morte. ${ }^{28}$ Segundo Perracini \& Ramos, ${ }^{10}$ idosos na faixa etária de 75 a 84 anos que precisam de auxílio nas atividades de vida diária possuem probabilidade 14 vezes maior de cair que idosos independentes.

Como relatado por outros autores, ${ }^{36} \mathrm{a}$ presença de sintomas sugestivos de depressão foi observada em grande parte das idosas que sofreram quedas (62\%). Entre as alterações emocionais, a depressão é a que mais influencia no risco de quedas, por predispor alterações no controle postural, de- sorientação visuo-espacial e distúrbios do comportamento associados. ${ }^{4}$ Diversos fatores podem precipitar a depressão na população idosa, como a presença de algumas comorbidades que cursam com disfunção cognitiva, doenças cardiovasculares e endocrinopatia, e o uso de medicações, entre outros. ${ }^{36} \mathrm{O}$ fato de, na velhice, a depressão vir associada com doenças orgânicas com maior frequência aumenta os níveis de incapacidade funcional e a propensão a quedas. ${ }^{\text {? }}$

A maior ocorrência de quedas no grupo de idosas que apresentou déficit cognitivo observada é respaldada pela literatura. Segundo Carvalho ${ }^{3}$ e Coutinho, ${ }^{4}$ o comprometimento da atenção e da memória recente, bem como apraxia, agnosia, desorientação espacial e deterioração das funções executivas, contribuem para maior ocorrência de quedas entre idosos.

\section{CONCLUSÃO}

O perfil do grupo de idosas inseridas no ambulatório do NAI da UnATI/UERJ que sofreram quedas no último ano é composto, em sua maioria, por mulheres com idade igual ou superior a 70 anos, com quatro ou mais co-morbidades e uso de até quatro medicações, que apresentaram disfunções nutricionais, déficit visual, mobilidade funcional alterada, sintomas sugestivos de depressão e de declínio cognitivo. Verificou-se maior número médio de quedas nas idosas que apresentaram comprometimento da capacidade funcional. 
Os resultados do presente estudo indicam, em concordância com o que vem sendo revisto na literatura, ${ }^{31}$ a necessidade de uma abordagem multidimensional para reduzir o risco de quedas em idosos, através de ações integradas e especializadas de uma equipe. Vale reforçar a importância de estimular e instrumentalizar os sujeitos para o autocuidado, assim como a família/cuidador, para que participem ativamente no controle das quedas.

A avaliação por meio dos testes funcionais, no momento da inserção do idoso no acompanhamento assistencial e em reavaliações periódicas programadas segundo um protocolo específico, pode evidenciar e monitorar, de forma simples e com baixo custo, alterações que demandem interven- ções terapêuticas preventivas, assistenciais e de reabilitação, que contribuam para uma maior expectativa de vida saudável e uma tentativa de recuperação ou manutenção da qualidade de vida de pessoas idosas que sofreram quedas. Essas informações são importantes, pois "a ocorrência de quedas no ano anterior, ainda que sem gravidade, é um importante sinalizador do risco de queda com fratura grave no ano seguinte".

Neste trabalho não foram contemplados fatores de risco ambientais e comportamentais que, sabidamente, têm papel importante no contexto das quedas. Sugere-se que estes sejam investigados na continuidade do acompanhamento assistencial das idosas, sobretudo as de maior risco, a fim de estimular ações preventivas que minimizem o risco de futuras quedas.

\section{REFERENCIAS}

1. Paixão Jr CM, Heckmann MF. Distúrbios da postura, marcha e quedas. In: Freitas EV, et al, organizadores. Tratado de Geriatria e Gerontologia. 2. ed. Rio de Janeiro: Guanabara Koogan, 2006. p. 950-60.

2. Instituto Brasileiro de Geografia e Estatística. Indicadores sociodemográficos prospectivos para o Brasil 1991-2030. Rio de Janeiro: IBGE. 2006. [Acesso 2008 jul. 28]. Disponível em: URL: < http://www.ibge.gov.br/home/ estatistica/populacao/ projecao_da_populacao/ publicacao_UNFPA.pdf $>$.

3. Brasil. Ministério da Saúde. Sistema de informações hospitalares do SUS (SIH/ SUS). Brasília: 2000. [Acesso 2008 ago 21]. Disponível em: URL: < http:// tabnet.datasus.gov.br/cgi/idb2007/ matriz.htm\#demog > .
4. Paschoal SMP, Lima EM. Quedas. In: Carvalho Filho ET, Papaléo Netto M. Geriatria. 2. ed. São Paulo: Atheneu, 2005. p. 581-90.

5. Pereira SRM, et al. Quedas em idosos. Rio de Janeiro: Sociedade Brasileira de Geriatria e Gerontologia, 2001.

6. Moura RN, et al. Quedas em idosos: fatores de risco associados. Gerontologia 1999; 7(2): 15-21.

7. Biderman A, et al. Depression and falls among community dwelling elderly people: a search for common risk factors. J Epidemiol Community Health 2002 aug; 56(8): 631-6.

8. Carvalho, AM. Demência como fator de risco para queda seguida de fratura grave em idosos [tese]. Rio de Janeiro: Fundação Oswaldo Cruz, Programa de 
Mestrado da Escola Nacional de Saúde Pública; 2000.

9. Coutinho ESF, Silva SD. Uso de medicamentos com fator de risco para fratura grave decorrente de queda em idosos. Caderno de Saúde Pública. 2002; 18(5):1359-66.

10. Perracini MR, Ramos RL. Fatores Associados a quedas em uma coorte de idosos residentes na comunidade. Revista de Saúde Pública 2002; 36(6): 709-16.

11. Rozenfeld S. Reações adversas aos medicamentos na terceira idade: as quedas como iatrogenia farmacoterapêutica [tese]. Rio de Janeiro: Instituto de Medicina Social, Programa de Doutorado da Universidade do Estado do Rio de Janeiro; 1997.

12. Lima-Costa MFF, et al. Diagnóstico da situação da população idosa brasileira: um estudo da mortalidade e das internações hospitalares públicas. Informe Epidemiológico do SUS 2000 mar; 9(1): 43-50.

13. Souza JAG, Iglesias ACRG. Trauma no idoso. Rev Assoc Med Bras 2002; 48(1): 79-86.

14. Stevens JA, et al. The costs of fatal and nonfatal falls among older adults. Inj Prev 2006; 12: 290-5.

15. Brasil. Ministério da Saúde. Política Nacional de Saúde da Pessoa Idosa. Brasília: 2006. [Acesso 2008 jul. 17]. Disponível em: URL: < http:// portal.saude.gov.br/portal/arquivos/pdf/ 2528\%20aprova\%20a\%20politica\%20nacional $\% 20$ de $\% 20$ saude $\% 20$ da\% 20pessoa\% 20idosa.pdf $>$.

16. Santos DM, Sichieri R. Body mass index and measures of adiposity among elderly adults. Rev Saúde Pública 2005 apr; 39(2): 163-8.

17. Runge PE. Eduard Jaeger's Test-Types (Schrift-Scalen) and the historical development of vision tests. Trans Am Ophthalmol Soc 2000; 98: 375-438.
18. Organização Mundial de Saúde (OMS). Envelhecimento ativo: uma política de saúde. Brasília: Organização PanAmericana de Saúde; 2005.

19. Lipschitz DA. Screening for nutritional status in the elderly. Prim Care 1994 mar; 21(1): 55-67.

20. Brasil. Ministério da Saúde. Secretaria de Atenção à Saúde. Departamento de Atenção Básica. Caderno de Atenção Básica: envelhecimento e saúde da pessoa idosa. Brasília, 2006. (19). [Acesso 2008 jul 28]. Disponível em: URL: < http:// dtr2004.saude.gov.br/dab/docs/ publicacoes/cadernos_ab/abcad19.pdf $>$.

21. Podsiadlo D, Richardson S. The timed "up and go": a test of basic functional mobility for frail elderly persons. J Am Geriatr Soc 1991; 39: 142-8.

22. Rockwood K, et al. Feasibility and measurement properties of the functional reach and the timed up and go tests in the Canadian study of health and aging. J Gerontol A Biol Sci Med Sci 2000 feb; 55(2): 70-3.

23. Katz S. Assessing self-maintenance: activities of daily living, mobility and instrumental activities of daily living. J Am Geriatr Soc 1983 dec; 31(12): 721-7.

24. Duarte YAO, Andrade CL, Lebrao ML. O Índex de Katz na avaliação da funcionalidade dos idosos. Revista da Escola de Enfermagem da USP 2007 jun; 41(2):317-25.

25. Paradela EMP, Lourenço RA, Veras RP. Validação da escala de depressão geriátrica em um ambulatório geral. Rev Saúde Pública 2005 dez; 39(6): 918-23.

26. Folstein MF, Folstein SE, McHugh PR. Mini-mental state: a practical method for grading the cognitive state of patients for clinician. J Psychiatr Res 1975; 12: 189-98.

27. Lourenço RA, Veras RP. Mini-exame do estado mental: características psicométricas em idosos ambulatoriais. Rev Saúde Pública 2006 ago; 40(4): 712-9. 
28. Vellas JB, et al. Malnutrition and falls. Lancet $1990 \mathrm{dez} ; 336: 1447$.

29. Anjos LA. Valores Antropométricos da População Adulta Brasileira: Resultados da Pesquisa Nacional sobre Saúde e Nutrição. [Tese]. Niterói: Universidade Federal Fluminense, 1993.

30. Menezes RL, Bachion MM. Estudo da presença de fatores de riscos intrínsecos para quedas, em idosos institucionalizados. Ciência \& Saúde Coletiva 2008 jul/ago; 13(4): 1209-18.

31. Tinetti, ME. Prevening falls in elderly persons. N Engl J Med 2003; 348: $42-9$.

32. Pereira LRL, et al. Avaliação da utilização de medicamentos em pacientes idosos por meio de conceitos de farmacoepidemiologia e farmacovigilância. Ciência \& Saúde Coletiva 2004; 9(2): 479-81.

33. Jack CIA, et al. Prevalence of low vision in elderly patients admitted to an acute geriatric unit in Liverpool: elderly people who fall are more likely to have low vision. Gerontology 1995; 41: 280-5.

34. Lee HKM, Scudds RJ. Comparison of balance in older people with and without visual impairment. Age Ageing 2003; 32(6): 643-9.

35. Vellas JB, et al. A two-year longitudinal study of falls in 482 community-dwelling elderly adults. J Gerontol A Biol Sci Med Sci 1998; 53: 264-74.

36. Turcu A, et al. Falls and depression in older people. Gerontology 2004 sept/oct; 50(5): 303-8. 